Research Article

\title{
Study of Magnetohydrodynamic Pulsatile Blood Flow through an Inclined Porous Cylindrical Tube with Generalized Time-Nonlocal Shear Stress
}

\author{
Nehad Ali Shah $\mathbb{D}^{1,2}$ A. Al-Zubaidi, ${ }^{3}$ and S. Saleem $\mathbb{D}^{3}$ \\ ${ }^{1}$ Informetrics Research Group, Ton Duc Thang University, Ho Chi Minh City, Vietnam \\ ${ }^{2}$ Faculty of Mathematics \& Statistics, Ton Duc Thang University, Ho Chi Minh City, Vietnam \\ ${ }^{3}$ Department of Mathematics, College of Science, King Khalid University, Abha 61413, Saudi Arabia \\ Correspondence should be addressed to Nehad Ali Shah; nehadali199@yahoo.com
}

Received 29 January 2021; Revised 23 April 2021; Accepted 6 May 2021; Published 18 May 2021

Academic Editor: Mustafa Inc

Copyright ( 2021 Nehad Ali Shah et al. This is an open access article distributed under the Creative Commons Attribution License, which permits unrestricted use, distribution, and reproduction in any medium, provided the original work is properly cited.

\begin{abstract}
The effects of pulsatile pressure gradient in the presence of a transverse magnetic field on unsteady blood flow through an inclined tapered cylindrical tube of porous medium are discussed in this article. The fractional calculus technique is used to provide a mathematical model of blood flow with fractional derivatives. The solution of the governing equations is found using integral transformations (Laplace and finite Hankel transforms). For the semianalytical solution, the inverse Laplace transform is found by means of Stehfest's and Tzou's algorithms. The numerical calculations were performed by using Mathcad software. The flow is significantly affected by Hartmann number, inclination angle, fractional parameter, permeability parameter, and pulsatile pressure gradient frequency, according to the findings. It is deduced that there exists a significant difference in the velocity of the flow at higher time when the magnitude of Reynolds number is small and large.
\end{abstract}

\section{Introduction}

The flow of blood into arteries is important in medical research. Computational blood flow simulation across vessels is one tool for integrating and interpreting clinical results. Specific hemodynamic flows may be predicted, which helps in disease detection. By deciding the form and design of blood vessels, it can also aid in the development of instruments that mimic or alter them. Blood movement in multistenosis arteries affected by pulsatile pressure gradient is one of the most difficult problems of fluid dynamics and biophysics. In the analysis that was carried out by Hatami et al. [1], blood was considered as a third grade non-Newtonian fluid conveying gold nanoparticles through a hollow porous vessel, and it was revealed that increase in the magnitude of the MHD (megnotohydrodynamics) parameter corresponds to a decrease in the velocity profile. The transient fluid dynamic equations of blood flow through stenosis geometry considering the non-Newtonian viscosity of blood and both magnetization and Lorentz forces was studied by
Amlimohamadi et al. [2]. They studied the real heart beating rate, the time-dependent inlet velocity alters, and the impact of the magnetic field on different heart cycles. In the presence of the external magnetic field, finite element simulation has been carried out by Alimohamadi and Imani [3] to invesigate the pulsatile blood flow through stenosed artery.

A simple theory that models the flow of a magnetohydrodynamic blood through pump can be found in Roberts [4], Korchevskii, and Marochnik [5] while an explicit scientific report on the influence of a magnetic field on blood flow, flow of blood in branched arteries, blood flow with periodic body acceleration, flow of blood in the collapsed of veins, impact of slip velocity factor on the flow of blood in the microcirculation, combined effects of curved boundary on the temperature distribution, metabolic heat production, and blood flow has been deliberated upon by [6-11]. Tzirtzilakis [12] investigated the mathematical model for blood flow in the presence of the magnetic field. In consistent with the principles of magnetohydrodynamics, ferrohydrodynamics, and by involving the electrical conductivity, Mekheimer 


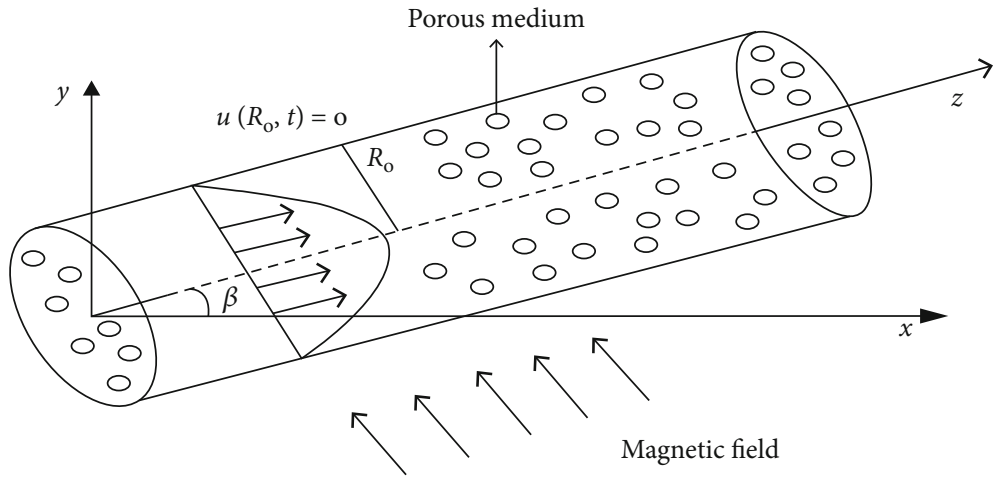

FIGURE 1: Schematic diagram of the flow geometry.

[13] discussed the influence of the uniform magnetic field on the peristaltic blood flow model. A mathematical governing model of blood flow in narrow and stenosis arteries under the influence of the magnetic field is presented by Jain et al. [14]. A numerical study of blood flow in stenosis tube due to the magnetic field is studied by Varshney and Gaurav [15]. Many other papers discussed blood flow models in stenosed arteries [16-19].

The effect of the magnetic field on parameters of blood in presence of magnetic particle through a circular tube is given by Sharma et al. [20]. The two-phase blood flow through a circular tube with magnetic properties has been studied by Zafar et al. [21]. He found the comparison of the analytical and semianalytical solutions of the classical model. The exact solutions of the blood flow model with fractional derivatives along with magnetic nanoparticles in the cylindrical domain have been found by Shah et al. [22].

Flow across a porous medium has a wide range of industrial applications. Blood flow through several stenosed porous arteries under the influence of a transverse magnetic field has been studied by Sinha et al. [23]. Magnetohydrodynamic MHD blood flow through porous vessel has been carried out by Ramamurthy and Shanker [24]. The peristaltic non-Newtonian Maxwell fluid flow through porous tube has been introduced by Eldesoky and Mousa [25].

In bioengineering, peristaltic blood flow in an inclined medium is a useful model. As a result, numerous studies of peristaltic blood flow models have been published. Through introducing a computational investigation of unsteady pulsatile blood flow through porous artery medium see [26]. Some important recent contributions to the mentioned topic are referenced in [27-30]. Generally, a fractional derivative model is obtained from the ordinary model by interchanging the derivatives of integer order with noninteger order.

Fractional dynamical systems demonstrate promise in the study of fluid flow models. In architecture and manufacturing, the fractional calculus approach has been used to obtain a useful generalization of physical concepts. Many students are interested in using fractional dynamics to solve problems in classical dynamics. However, Riemann-Liouville and Caputo fractional derivatives are commonly used, and this generalization can be done by using different other fractional approaches/definitions $[31,32]$.
Many models used fractional calculus to solve fluid flow problems [33, 34]. In the year 2016, Shah et al. [22] used Caputo-Fabrizio derivative to obtain the exact solutions of pulsatile blood flow in a circular cylinder. In the study, Laplace and Hankel transform was successfully used to further solve the momentum and energy equation. In that study, the influence of MHD, porous medium, and inclined surface was ignored. Motivating by Shah et al. [28], we have obtained the analytic and semianalytical solutions of unsteady MHD blood flow through an inclined porous tube that has been studied in the presence of peristaltic pressure gradient. The analysis is made by employing Laplace transformation method, and some valuable predictions have been carried out from the study. For the semianalytic solution, the inverse Laplace transform has been calculated by using numerical package though Mathcad because the velocity expressions of Laplace transform are in the complex form of modified Bessel functions. Therefore, it is almost impossible to find inverse Laplace analytically. To show the accuracy of our obtained results of inverse Laplace transform, these results are compared with two other inverse Laplace transform numerical algorithms, named as Stehfest's [35] and Tzou's [36] algorithms. Finally, the effect of pertinent physical parameters is discussed in detail.

\section{Formulation of the Problem}

Consider an inclined tapered axisymmetric cylindrical tube of radius $R_{0}$ with an unsteady pulsatile blood flow in a porous medium.

Figure 1 shows how a fluid subjected to a uniform transverse magnetic field behaves in a perpendicular direction to the tube. The induced magnetic field as well as the external electric field is not taken into account. The cylindrical coordinate system $(r, \theta, z)$ is introduced with the $z$-axis that lies along the center of the artery and $r$ transverse to it. The unsteady magneto hydrodynamic incompressible flow of blood through an inclined tapered artery defined by following governing equations:

$$
\begin{aligned}
& \nabla \cdot \vec{V}=0, \\
& \rho \frac{D \vec{V}}{D t}=\nabla \cdot T-\frac{\mu \vec{V}}{k}+J \times B+\rho g \sin \beta,
\end{aligned}
$$




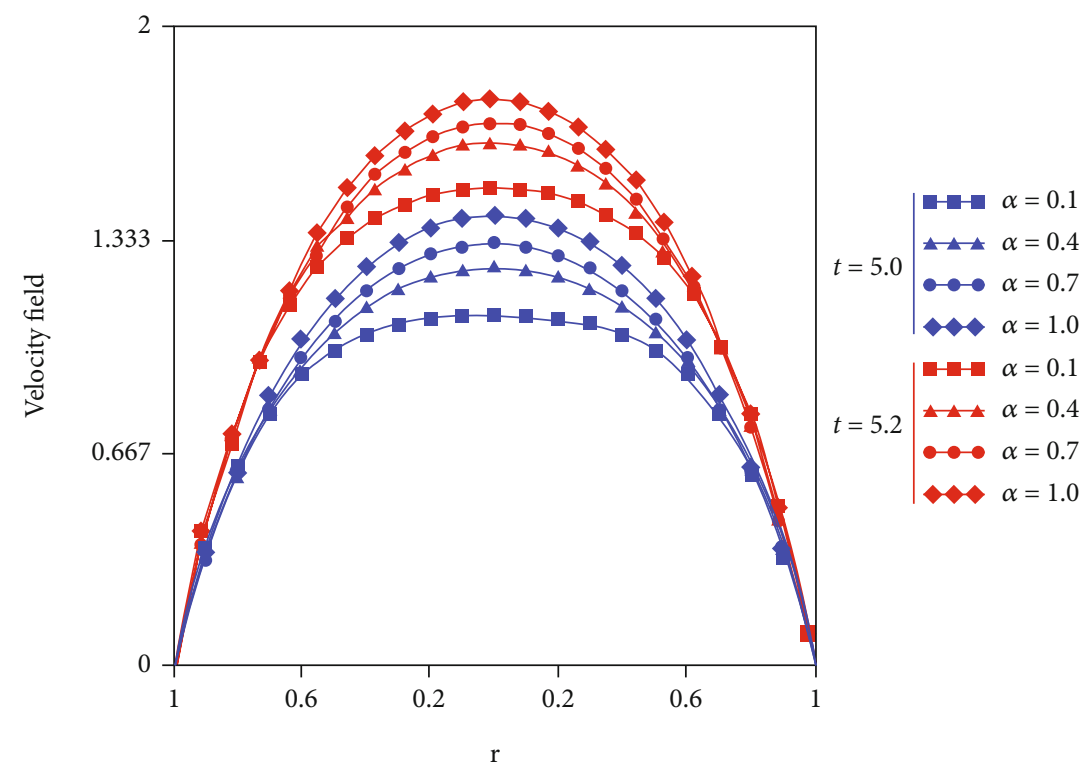

(a)

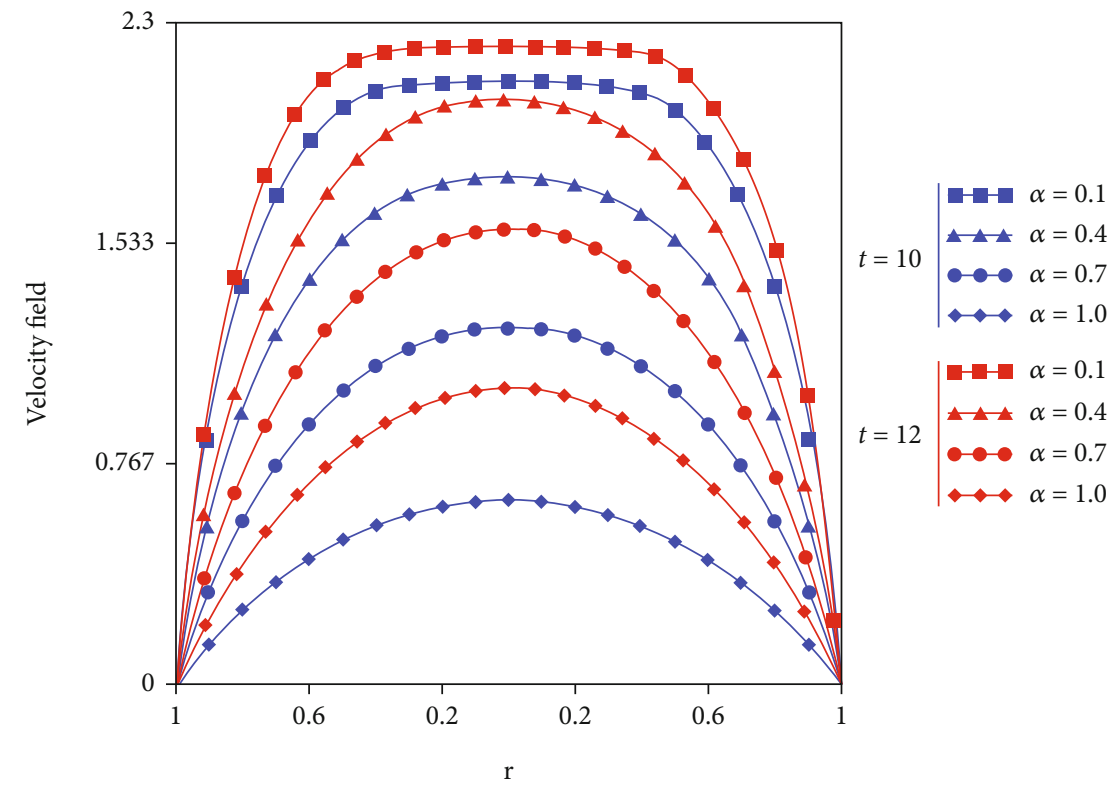

(b)

FIgURe 2: Profiles of dimensionless velocity for $\alpha$ versus $r$ at $\operatorname{Re}=2.5, \mathrm{Ha}=0.5, K=1, n=0.8$, for small and large values of time $t$.

where $\vec{V}$ is the velocity vector of the fluid, $\rho$ is the fluid density, $D / D t$ is the material time derivative, and $g$ is the external body force. Maxwell equations are written as

$$
\nabla \cdot \vec{B}=0, \nabla \times \vec{B}=\mu_{0} \vec{J}, \nabla \times \vec{E}=-\frac{\partial \vec{B}}{\partial t}
$$

where $\mu_{0}$ is the magnetic permeability, $\vec{J}$ is the current density, $\vec{B}$ is the magnetic field, and $\vec{E}$ is the electric field. The electric current density can be written by Ohm's law as [37]

$$
\vec{J}=\sigma(\vec{E}+\vec{V} \times \vec{B})
$$

where $\sigma$ is the electric conductivity. The electromagnetic force $F_{\text {emag }}$ can be expressed as

$$
\vec{F}_{\text {emag }}=\vec{J} \times \vec{B}=\sigma(\vec{E}+\vec{V} \times \vec{B}) \times \vec{B}=-\sigma B_{0}^{2} \omega(r, t) \vec{k},
$$

where $\vec{k}$ is the unit vector in the $z$ direction, and $\vec{V}=u(r, t) \vec{k}$ is the velocity of the blood along the axis of the cylindrical tube. The governing equation of the motion for flow in cylindrical polar coordinates $[20-22,38]$ is given by

$$
\begin{aligned}
\rho \frac{\partial u(r, t)}{\partial t}= & -\frac{\partial p}{\partial z}+\frac{1}{r} \frac{\partial r \tau_{z r}(r, t)}{\partial r}-\frac{\mu}{k} u(r, t) \\
& -\sigma B_{0}^{2} u(r, t)+\rho g \sin \beta,
\end{aligned}
$$




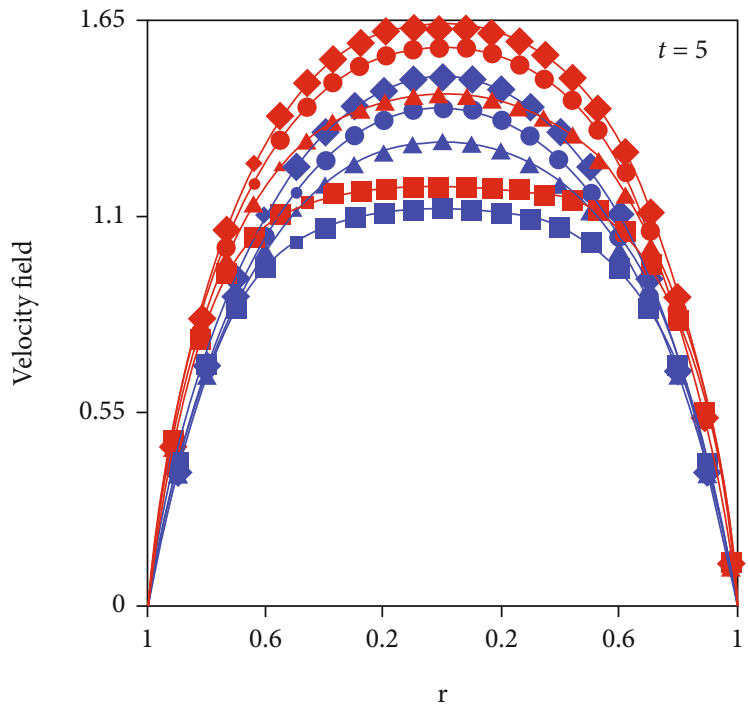

(a)

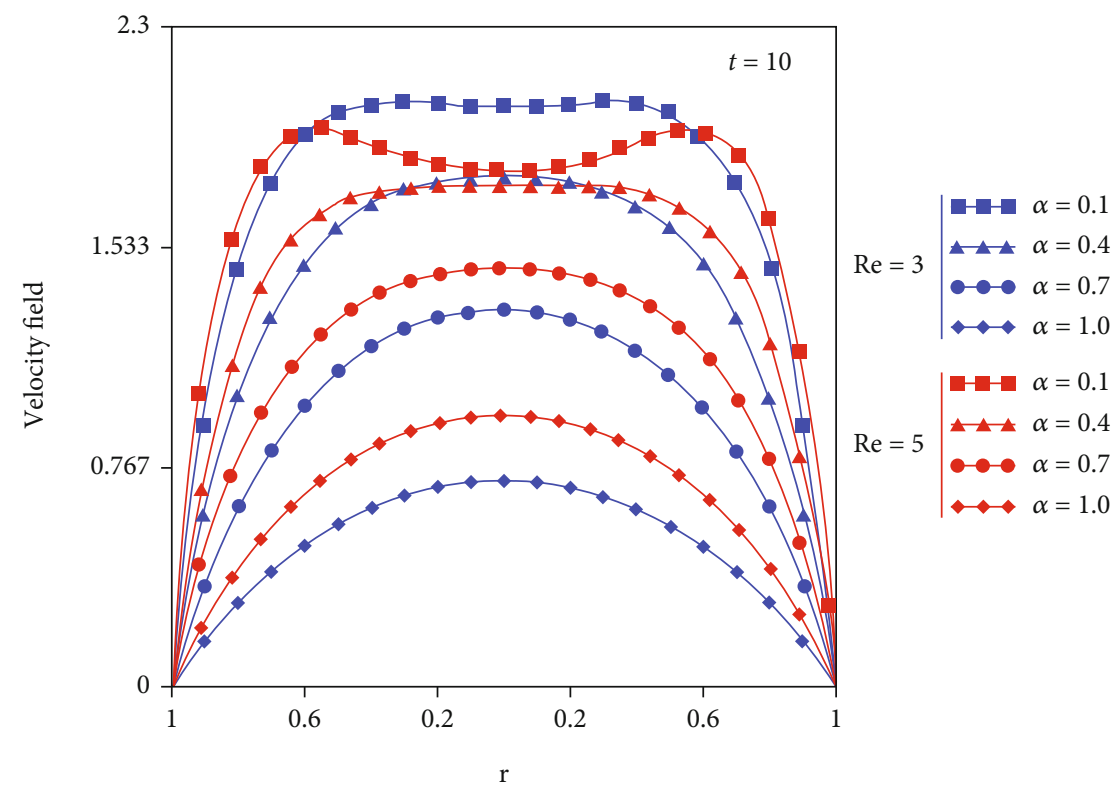

(b)

Figure 3: Profiles of dimensionless velocity for $\alpha$ versus $r$ at $\operatorname{Re}=3,5, \mathrm{Ha}=0.5, K=1, n=0.8$, for small and large values of time $t$.

$$
\tau_{r z}(r, t)=\mu \frac{\partial u(r, t)}{\partial r}
$$

where the pressure gradient of the form as $-\partial p / \partial z=S_{0}+S_{1}$ $\cos (\xi t), S_{0}$ and $S_{1}$ are amplitudes of pulsatile systolic or diastolic pressure gradient, and $\xi$ is the frequency of the pulse. The above model becomes also that we can write

$$
\begin{aligned}
\rho \frac{\partial u(r, t)}{\partial t}= & S_{0}+S_{1} \cos (\xi t)+\frac{1}{r} \frac{\partial r \tau_{z r}(r, t)}{\partial r}-\frac{\mu}{k} u(r, t) \\
& -\sigma B_{0}^{2} u(r, t)+\rho g \sin \beta .
\end{aligned}
$$

The boundary conditions that must be satisfied by the blood on the wall of artery are following

$$
u(r, 0)=0, u\left(R_{0}, t\right)=0,\left.\frac{\partial u(r, t)}{\partial r}\right|_{r=0}=0
$$

Let us introduce the following dimensionless variables:

$$
r^{*}=\frac{r}{R_{0}}, t^{*}=\frac{t}{t_{0}}, u^{*}=\frac{u}{u_{0}}, g^{*}=\frac{g t_{0}}{u_{0}}, S_{0}^{*}=\frac{t_{0} S_{0}}{\rho u_{0}}, S_{1}^{*}=\frac{t_{0} S_{1}}{\rho u_{0}}, \tau^{*}=\frac{R_{0} \tau}{\mu u_{0}},
$$




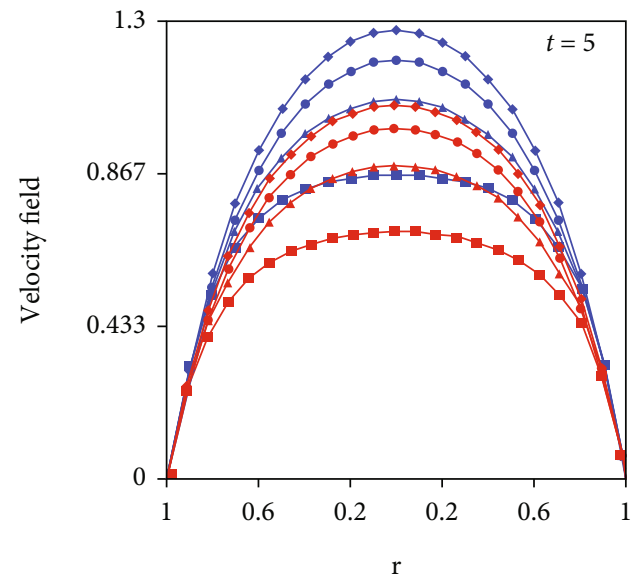

(a)

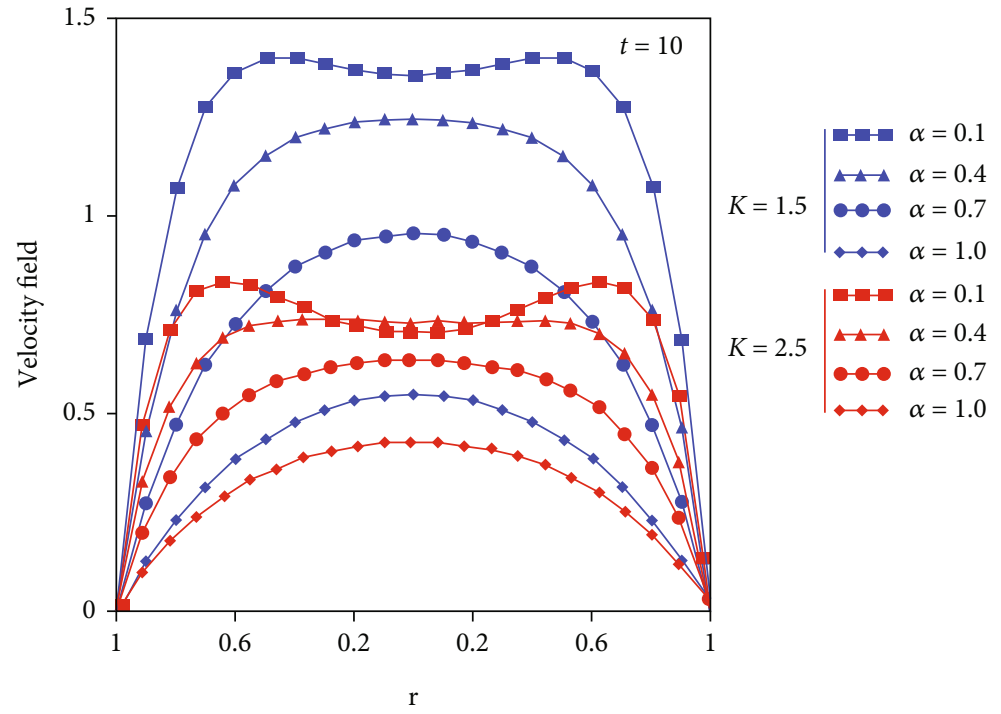

(b)

Figure 4: Profiles of dimensionless velocity for $\alpha$ versus $r$ at $\mathrm{Re}=2.5, \mathrm{Ha}=0.5, K=1.5,2.5, n=0.8$, for small and large values of time $t$.

where $u_{0}$ is the characteristic velocity, and $t_{0}$ is the characteristic time. Using the above dimensionless variables and parameters and after dropping out the $*$ notation in Eqs. (6), (7), and (8), we obtain

$$
\begin{aligned}
& \frac{\partial u(r, t)}{\partial t}= S_{0}+S_{1} \cos (\xi t)+\frac{1}{\operatorname{Re}} \frac{1}{r} \frac{\partial r \tau_{z r}(r, t)}{\partial r} \\
&-K u(r, t)-H a^{2} u(r, t)+m, \\
& \tau_{r z}(r, t)= \frac{\partial u(r, t)}{\partial r}, \\
& u(r, 0)=0, u(1, t)=0,\left.\frac{\partial u(r, t)}{\partial r}\right|_{r=0}=0,
\end{aligned}
$$

where $H a=B_{0} \sqrt{t_{0} v \sigma / \mu}$ represents the Hartmann number, $\operatorname{Re}=R_{0}{ }^{2} / v t_{0}$ describe the Reynolds number, and $m=t_{0} g$ $\sin \beta / \mu$ is the inclination parameter.

In the following, we develop a fractional model in which the classical constitutive Eqs. (9) and (11) are generalized by using the constitutive shear stress equation

$$
\tau_{z r}(r, t)={ }^{C} D_{t}^{1-\alpha}\left(\frac{\partial u(r, t)}{\partial y}\right) ; 0<\alpha \leq 1
$$

proposed by Scott-Blair [39]. The Caputo fractional derivative formula of order $\alpha$ is defined as [40]

$$
D_{t}^{\alpha} u(y, t)= \begin{cases}\frac{1}{\Gamma(1-\alpha)} \int_{0}^{t} \frac{1}{(t-\tau)^{\alpha}} \frac{\partial u(y, \tau)}{\partial \tau} d \tau ; & 0<\alpha<1, \\ \frac{\partial u(y, t)}{\partial t} ; & \alpha=1,\end{cases}
$$

where $\Gamma$ denotes the Gamma function.
Using Eq. (12) in Eq. (10), we obtain

$$
\begin{aligned}
\frac{\partial u(r, t)}{\partial t}= & S_{0}+S_{1} \cos (\xi t)+\frac{1}{\operatorname{Re}}{ }^{C} D^{1-\alpha}\left(\frac{\partial^{2} u(r, t)}{\partial r^{2}}+\frac{1}{r} \frac{\partial u(r, t)}{\partial r}\right) \\
& -K u(r, t)-H a^{2} u(r, t)+m .
\end{aligned}
$$

\section{Analytical Solution}

Taking Laplace transformation of Eq. (13), we obtain

$$
\begin{aligned}
q \bar{u}(r, q)= & \frac{S_{0}}{q}+\frac{S_{1} q}{q^{2}+\xi^{2}}+\frac{1}{\operatorname{Re}} q^{1-\alpha}\left(\frac{\partial^{2} \bar{u}(r, q)}{\partial r^{2}}+\frac{1}{r} \frac{\partial \bar{u}(r, q)}{\partial r}\right) \\
& -K \bar{u}(r, q)-H a^{2} \bar{u}(r, q)+\frac{m}{q} . .
\end{aligned}
$$

Applying finite Hankel transform to Eq. (16) and using initial and boundary conditions in Eq. (12), we obtain

$$
\begin{aligned}
\bar{u}_{H}\left(r_{n}, q\right)= & \frac{J_{1}\left(r_{n}\right)}{r_{n}}\left(\frac{S_{0}}{q}+\frac{S_{1} q}{q^{2}+\xi^{2}}+\frac{m}{q}\right) \\
& \cdot \frac{1}{q+\left(r_{n}^{2} / \mathrm{Re}\right) q^{1-\alpha}+K+H a^{2}},
\end{aligned}
$$

where $\bar{u}_{H}\left(r_{n}, q\right)=\int_{0}^{1} \bar{u}(r, q) r J_{0}\left(r r_{n}\right) d r$ is the finite Hankel transform of function $\bar{u}(r, q)$ and $r_{n}, n=1,2 \cdots$ is the positive roots of the equation $J_{0}(x)=0$, and $J_{0}$ being the Bessel function of the first kind and order zero. 


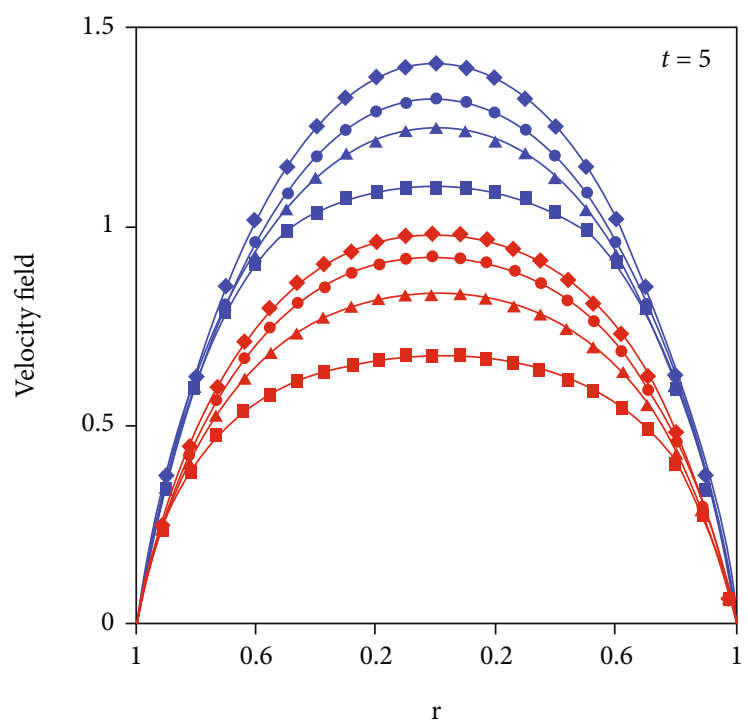

(a)

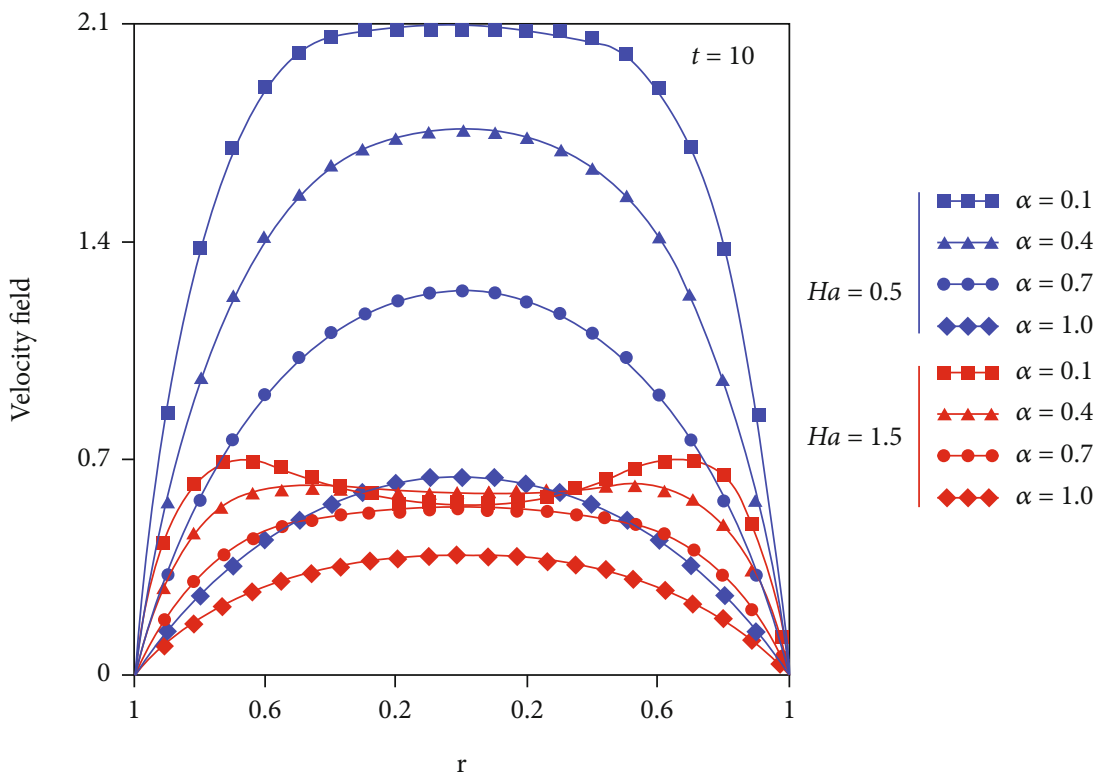

(b)

Figure 5: Profiles of dimensionless velocity for $\alpha$ versus $r$ at $\mathrm{Re}=2.5, \mathrm{Ha}=0.5,1.5, K=1, n=0.8$, for small and large values of time $t$.

Using the series formula $1 / z+a=\sum_{k=0}^{\infty}(-1)^{k} z^{k} / a^{k+1} ;|z|$ $a \mid<1$, Eq. (17) can be written as

$\bar{u}_{H}\left(r_{n}, q\right)=\frac{J_{1}\left(r_{n}\right)}{r_{n}}\left(\frac{S_{0}}{q}+\frac{S_{1} q}{q^{2}+\xi^{2}}+\frac{m}{q}\right) \sum_{k=0}^{\infty} \frac{(-1)^{k}\left(r_{n}^{2} / \mathrm{Re}\right)^{k} q^{k-\alpha k}}{\left(q+K+H a^{2}\right)^{k+1}}$.

Applying inverse Laplace transform to Eq. (18), we obtain

$$
\begin{aligned}
u_{H}\left(r_{n}, t\right)= & \frac{J_{1}\left(r_{n}\right)}{r_{n}}\left(S_{0}+S_{1} \cos (\xi t)+m\right) \\
& * \sum_{k=0}^{\infty}(-1)^{k}\left(\frac{r_{n}^{2}}{\mathrm{Re}}\right)^{k} G_{1, k-\alpha k, k+1}\left(K+H a^{2}, t\right),
\end{aligned}
$$

here $*$ represents the convolution product and

$$
G_{a, b, c}(d, t)=L^{-1}\left\{\frac{p^{b}}{\left(p^{a}-d\right)^{c}}\right\}, R(p)>0, R(a c-b)>0,\left|\frac{d}{p^{a}}\right|<1,
$$

is the generalized G-function of Lorenzo and Hartley [41].

Taking inverse Hankel transform of Eq. (19), we obtain

$$
\begin{aligned}
u(r, t)= & 2 \sum_{n=1}^{\infty} \frac{J_{0}\left(r r_{n}\right)}{J_{1}^{2}\left(r_{n}\right)} u_{H}\left(r_{n}, t\right) \\
= & 2 \sum_{n=1}^{\infty} \frac{J_{0}\left(r r_{n}\right)}{r_{n} J_{1}\left(r_{n}\right)}\left(S_{0}+S_{1} \cos (\xi t)+m\right) \\
& * \sum_{k=0}^{\infty}(-1)^{k}\left(\frac{r_{n}^{2}}{\operatorname{Re}}\right)^{k} G_{1, k-\alpha k, k+1}\left(K+H a^{2}, t\right) .
\end{aligned}
$$




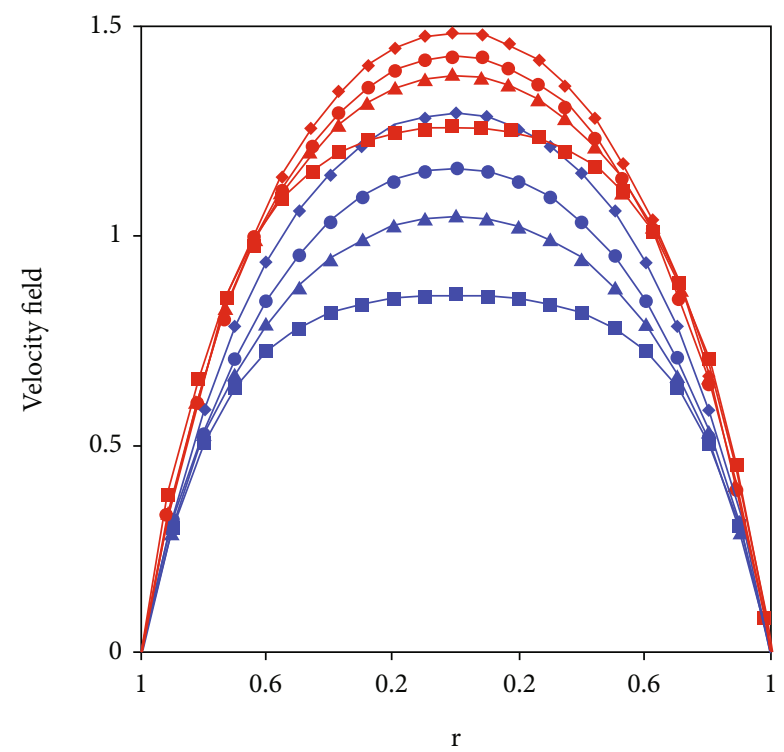

(a)

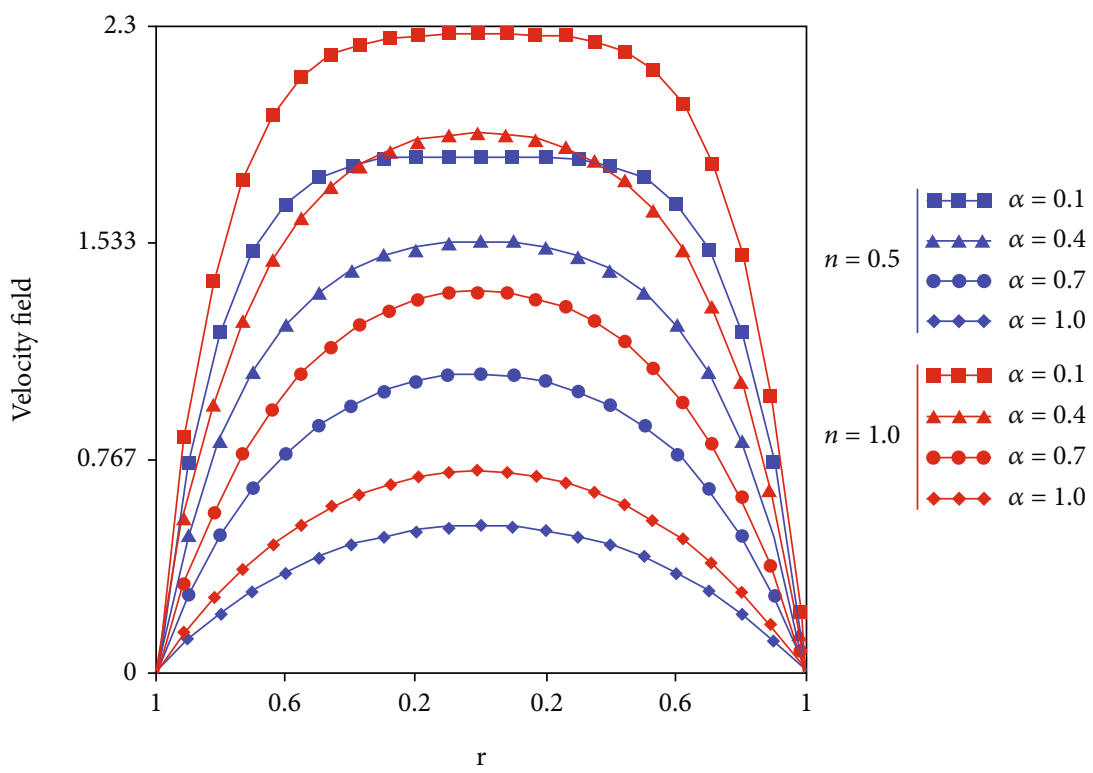

(b)

Figure 6: Profiles of dimensionless velocity for $\alpha$ versus $r$ at $\operatorname{Re}=2.5, \mathrm{Ha}=0.5, K=1, n=0.5,1$, for small and large values of time $t$.

\section{Semianalytical Solution}

Taking Laplace transformation of Eq. (13), we obtain

$$
\begin{aligned}
q \bar{u}(r, q)= & \frac{S_{0}}{q}+\frac{S_{1} q}{q^{2}+\xi^{2}}+\frac{1}{\operatorname{Re}} q^{1-\alpha}\left(\frac{\partial^{2} \bar{u}(r, q)}{\partial r^{2}}+\frac{1}{r} \frac{\partial \bar{u}(r, q)}{\partial r}\right) \\
& -K \bar{u}(r, q)-H a^{2} \bar{u}(r, q)+\frac{m}{q} .
\end{aligned}
$$

By rearranging Eq. (22), we have

$$
\frac{\partial^{2} \bar{u}(r, q)}{\partial r^{2}}+\frac{1}{r} \frac{\partial \bar{u}(r, q)}{\partial r}-A(q) \bar{u}(r, q)=B(q)
$$

where $\quad A(q)=\operatorname{Re}\left(K q^{\alpha-1}+H a^{2} q^{\alpha-1}+q^{\alpha}\right)$ and $B(q)=-\operatorname{Re}$ $\left(\left(m q^{\alpha} / q^{2}\right)+\left(S_{0} q^{\alpha} / q^{2}\right)+\left(S_{1} q^{\alpha} / q^{2}+\xi^{2}\right)\right)$.

The solution of above nonhomogeneous second order differential Eq. (23) by using the initial and boundary conditions (12) in the transform domain is written as

$$
\bar{u}(r, q)=\frac{B(q)}{A(q)}\left[\frac{I_{0}(r \sqrt{A(q)})}{I_{0}(\sqrt{A(q)})}-1\right] .
$$

By writing $\bar{u}(y, q)$ in suitable and simple form, we can determine its inverse Laplace transform traditionally but Eq. (24) is in a complex form of the modified Bessel function, and it is not easy to use for some practical applications. 
TABLE 1: Comparison of two Laplace inverse numerical algorithms with analytical solution.

\begin{tabular}{lccccc}
\hline $\mathrm{r}$ & $u(r, t)$ [Eq. (21)] & $u_{S}(r, t)$ [Stehfest's] & $u_{T}(r, t)[$ Tzou's] & $\left|u(r, t)-u_{S}(r, t)\right|$ & 0.415058 \\
\hline 0 & 0.684151 & 1.099209 & 0.827544 & 0.293739 & 0.143394 \\
0.1 & 0.802388 & 1.096127 & 0.82701 & 0.338683 & 0.024621 \\
0.2 & 0.747556 & 1.086239 & 0.824787 & 0.276079 & 0.077232 \\
0.3 & 0.791451 & 1.06753 & 0.818938 & 0.291901 & 0.027487 \\
0.4 & 0.74445 & 1.036351 & 0.805933 & 0.199542 & 0.061483 \\
0.5 & 0.787414 & 0.986957 & 0.780184 & 0.17615 & $1.318152 e-3$ \\
0.6 & 0.734645 & 0.910795 & 0.733327 & $5.161311 \mathrm{e}-3$ & 0.137146 \\
0.7 & 0.790328 & 0.79549 & 0.653183 & 0.083288 & 0.184388 \\
0.8 & 0.706713 & 0.623425 & 0.522325 & 0.397913 & 0.451604 \\
0.9 & 0.767713 & 0.3698 & 0.316108 & $1.479346 e-7$ & 0 \\
1 & 0 & $1.479346 \times 10^{-7}$ & 0 & \\
\hline
\end{tabular}

Moreover, the numerical Laplace method is considerd as an effective tool in computing the fractional differential equations. Sheng et al. [42] reported that the numerical inverse Laplace transform algorithms are efficacious and reliable for fractional-order differential equations. Stehfest's algorithm [31] successfully used by Tong et al. [43] and Jiang et al. [44]. Therefore, in this work, we apply the numerical algorithm of the inverse Laplace transform method to Eq. (24) and analyze the flow properties. Stehfest's formula is defined as

$$
u(r, t)=\frac{\ln (2)}{t} \sum_{j=1}^{2 m} d_{j} \bar{u}\left(r, j \frac{\ln (2)}{t}\right)
$$

where $m$ is a positive integer.

$$
d_{j}=(-1)^{j+m} \sum_{i=\left[\frac{j+1}{2}\right]}^{\min (j, m)} \frac{i^{m}(2 n) !}{(m-i) ! i !(i-1) !(j-i) !(2 i-j) !},
$$

and $[r]$ denotes the integer value function or bracket function.

Tzou's formula can be defined as

$u(r, t)=\frac{e^{4.7}}{t}\left[u\left(r, \frac{4.7}{t}\right)+\operatorname{Re}\left[\sum_{j=1}^{\infty}(-1)^{n} u\left(r, \frac{4.7+j \pi i}{t}\right)\right]\right]$

The numerical solutions of transformed Eq. (24) have been obtained by using algorithms (25) and (27), and results are presented in tables.

\section{Numerical Results and Discussion}

In this section, the analyses of physical parameters on the fluid flow are presented as Figures 2-6.

In Figure 2, we present the effect of the fractional parameter $\alpha$ for different small and large values of time $t$. From Figure 2(a), it is observed that for a small value of time, the ordinary fluid velocity is maximum than fractional fluid flow. While by increasing the value of time, the fluid flow velocity decreases. In Figure 2(b), the influence is opposite than Figure 2(a), for a large value of time. A further attempt was made to quantify the effect on the velocity profile by using the slope of linear regression through the data points as presented by Animasaun and Pop [45]. In between the artery $(-0.2 \leq r \leq 0.2)$, the optimal effect is seen when $t=5.0$ the slope of regression line through the data in known velocity and the order of Caputo fractional derivatives is -0.3173000 . When $t=5.2$, a decrease in the velocity field is also noticed, and the rate is estimated using the same approach as -0.2705067 . Due to the singular kernel of the fractional derivative for small values and large values of the time $t$, the flow has opposite influence. It is worthy to note that the effects of fractional parameter $\alpha$ as reported in this study complement that of ref. [46] in which heat transfer in the flow of a fractional viscous fluid over an infinite vertical plate with exponential heating using a fractional derivative with nonsingular kernel is deliberated upon. Consecutively, the effect of Reynolds number Re on the flow is presented in Figure 3, for small and large values of time. It is deduced that there exists a significant difference between the flow when $t=5, \operatorname{Re}=3$ and $t=5, \operatorname{Re}=5$. At the initial time $(t=5)$, it is seen that the velocity of the flow increases with the order of Caputo fractional derivative when $\mathrm{Re}=3$ and $\operatorname{Re}=5$, see Figure 3(a). It is worth pointing out that the maximum velocity field is obtained at larger values of Reynolds number. At larger values of time, it is interesting to reveal that a decrease in the velocity field is guaranteed, see Figure $3(\mathrm{~b})$. When $\operatorname{Re}=3$, the slope of regression line through the data in known velocity and the order of Caputo fractional derivatives is -0.4527000 . However, when $\operatorname{Re}=5$, the rate of decrease in the velocity field is quantified using the slope of regression line as -0.7884667 .

The effect of the porous parameter $K$ is presented in Figure 4, for small and large values of time. For a small time, it is seen that the velocity increases due to an increase in the magnitude of the porous parameter $K$. For large values of time, interesting results found that in the medium of the cylinder for fractional parameter less than 0.7 by increasing $K$, the velocity increases on the other hand, and for fractional parameter greater than 0.7 by increasing $K$, the velocity decreases. Figure 5 reveals the influence of Hartman number 
TABLE 2: Effect of noninteger order of fractional parameter on the velocity field.

\begin{tabular}{lccccc}
\hline$\alpha$ & $u(r, t)$ [Eq. (21)] & $u_{S}(r, t)$ [Stehfest's] & $u_{T}(r, t)$ [Tzou's] & $\left|u(r, t)-u_{S}(r, t)\right|$ & $\left|u(r, t)-u_{T}(r, t)\right|$ \\
\hline 0 & 0.659024 & 0.986618 & 0.769764 & 0.327593 & 0.11074 \\
0.1 & 0.74445 & 1.036351 & 0.805933 & 0.291901 & 0.061483 \\
0.2 & 0.814984 & 1.074099 & 0.831608 & 0.259115 & 0.016623 \\
0.3 & 0.872347 & 1.102689 & 0.849336 & 0.230342 & 0.023011 \\
0.4 & 0.918044 & 1.124687 & 0.861515 & 0.206643 & 0.056529 \\
0.5 & 0.953435 & 1.142488 & 0.870428 & 0.189053 & 0.083007 \\
0.6 & 0.979774 & 1.158389 & 0.878262 & 0.178615 & 0.101513 \\
0.7 & 0.998231 & 1.174669 & 0.887155 & 0.176438 & 0.111077 \\
0.8 & 1.009872 & 1.19366 & 0.899271 & 0.183787 & 0.110601 \\
0.9 & 1.01565 & 1.217833 & 0.916927 & 0.202184 & 0.098723 \\
1 & 1.016406 & 1.249903 & 0.942799 & 0.233496 &
\end{tabular}

$H a$. It is observed that by increasing the value of $\mathrm{Ha}$, the velocity of the fluid is found to be an increasing function when $t=5$ and a decreasing function when $t=10$. Physically, the negligible increasing effect in the velocity field can be traced to the fact that the effects of Lorentz force have not been fully materialized at initial time. The effect of the inclination parameter $n$ is represented in Figure 6. It is clear from Figure 6(a) by increasing the value of $n$ the velocity increases by considering the small value of time $t$. By increasing the value of time $t=10$, the influence of $n$ is much more.

In Tables 1 and 2, we make a comparison between analytical solution in Eq. (21) with numerical algorithms, named as Stehfest's [35] and Tzou's [36] algorithms. It is found that the analytical solution in Eq. (21) is in a good agreement with Tzou's algorithm.

\section{Conclusion}

The effect of a uniform magnetic field on unsteady blood flow through a peristaltic pressure gradient in an inclined porous tube has been investigated. The solution was discovered using the Laplace transformation technique, and the analysis yielded some useful predictions. For the semianalytical solution, the inverse Laplace transform has been calculated by using numerical package though Mathcad, since the velocity expressions of Laplace transform are in the complex form of modified Bessel functions. Therefore, it is very difficult to find inverse Laplace analytically. To show the accuracy of our obtained result, the result was compared with two other inverse Laplace transform numerical algorithms, named as Stehfest's [33] and Tzou's [34] algorithms. The effects of pertinent physical parameters are discussed in detail. These are some main results of the study:

(1) For small values of time, the fractional parameter $\alpha$ is inversely proportional to the velocity field, and it shows an opposite behavior for greater values of time

(2) There exists a significant difference in the velocity of the flow at a higher time when the magnitude of Reynolds number is small and large
(3) The effect of porous permeability and inclination is opposite to the velocity field as compared to the magnetic field. By increasing these parameters, the higher velocity field is ascertained

(4) Hartman number has dual effects on the velocity of the flow due to the fact that the impact of Lorentz force at the initial time is infinitesimal

(5) By comparing the analytical solution in Eq. (21) with numerical algorithms, named as Stehfest's and Tzou's algorithms, it is found that the analytical solution in Eq. (21) is in a good agreement with Tzou's algorithm

\section{Data Availability}

The data used to support the findings of this study are available from the corresponding author upon request.

\section{Conflicts of Interest}

The authors declare that they have no conflicts of interest.

\section{Acknowledgments}

The authors extend their appreciation to the Deanship of Scientific Research at King Khalid University for funding this work through research group program under Grant No. RGP.1/324/42.

\section{References}

[1] H. Hatami, J. Hatami, and D. D. Ganji, "Computer simulation of MHD blood conveying gold nanoparticles as a third grade non-Newtonian nanofluid in a hollow porous vessel," Computer Methods and Programs in Biomedicine, vol. 113, no. 2, pp. 632-641, 2014.

[2] H. Amlimohamadi, M. Akram, and K. Sadeghy, "Flow of a Casson fluid through a locally-constricted porous channel: a numerical study," Korea-Australia Rheology Journal, vol. 28, no. 2, pp. 129-137, 2016.

[3] H. Alimohamadi and M. Imani, "Finite element simulation of two-dimensional pulsatile blood flow through a stenosed 
artery in the presence of external magnetic field," International Journal for Computational Methods in Engineering Science and Mechanics, vol. 15, no. 4, pp. 390-400, 2014.

[4] V. C. Roberts, "Magnetohydrodynamic pumping of blood," Medical and Biological Engineering and Computing, vol. 10, no. 1, pp. 57-59, 1972.

[5] M. Korchevskii and L. S. Marochnik, "Magnetohydrodynamic version of movement of blood," Biophysics, vol. 10, pp. 411414, 1965.

[6] V. A. Vardanyan, "Effect of a magnetic field on blood flow," Biophysics, vol. 18, pp. 515-521, 1973.

[7] P. K. Suri and R. Suri Pushpa, "Blood flow in a branched-arteries," Indian Journal of Pure and Applied Mathematics, vol. 12, pp. 907-918, 1981.

[8] P. Chaturani and V. Palanisamy, "Pulsatile flow of blood with periodic body acceleration," International Journal of Engineering Science, vol. 29, no. 1, pp. 113-121, 1991.

[9] K. W. Chow and C. C. Mak, "A simple model for the two dimensional blood flow in the collapse of veins," Journal of Mathematical Biology, vol. 52, no. 6, pp. 733-744, 2006.

[10] D. Pal, N. Rudraiah, and R. Devanathan, "The effects of slip velocity at a membrane surface on blood flow in the microcirculation," Journal of Mathematical Biology, vol. 26, no. 6, pp. 705-712, 1988.

[11] D. F. Jamil, S. Saleem, R. Roslan et al., "Analysis of nonNewtonian magnetic Casson blood flow in an inclined Stenosed artery using Caputo-Fabrizio fractional derivatives," Computer Methods and Programs in Biomedicine, vol. 203, article I106044, p. 106044, 2021.

[12] E. E. Tzirtzilakis, "A mathematical model for blood flow in magnetic field," Physics of Fluids, vol. 17, no. 7, article 077103, 2005.

[13] K. S. Mekheimer, "Peristaltic flow of blood under effect of a magnetic field in a non-uniform channels," Applied Mathematics and Computation, vol. 153, no. 3, pp. 763-777, 2004.

[14] M. Jain, G. C. Sharma, and A. Singh, "Mathematical analysis of MHD flow of blood in very narrow capillaries," Inter. J. Eng. Transactions B, vol. 22, pp. 307-315, 2009.

[15] V. K. G. Varshney, V. Katiyar, and S. Kumar, "Effect of magnetic field on the blood flow in artery having multiple stenosis: a numerical study," International Journal of Engineering, Science and Technology, vol. 2, no. 2, pp. 67-82, 2010.

[16] K. S. Mekheimer, T. Elnaqeeb, M. A. El Kot, and F. Alghamdi, "Simultaneous effect of magnetic field and metallic nanoparticles on a micropolar fluid through an overlapping stenotic artery: blood flow model," Physics Essays, vol. 29, no. 2, pp. 272-283, 2016.

[17] T. Elnaqeeb, K. S. Mekheimer, and F. Alghamdi, "Cu-blood flow model through a catheterized mild stenotic artery with a thrombosis," Mathematical Biosciences, vol. 282, pp. 135146, 2016.

[18] T. Elnaqeeb, N. A. Shah, and K. S. Mekheimer, "Hemodynamic characteristics of gold nanoparticle blood flow through a tapered stenosed vessel with variable nanofluid viscosity," BioNanoScience, vol. 9, no. 2, pp. 245-255, 2019.

[19] T. Elnaqeeb, "Modeling of au (NPs)-blood flow through a catheterized multiple stenosed artery under radial magnetic field," The European Physical Journal Special Topics, vol. 228, no. 12, pp. 2695-2712, 2019.

[20] S. Sharma, U. Singh, and V. K. Katiyar, "Magnetic field effect on flow parameters of blood along with magnetic particles in a cylindrical tube," Journal of Magnetism and Magnetic Materials, vol. 377, pp. 395-401, 2015.

[21] A. A. Zafar, N. A. Shah, and I. Khan, "Two phase flow of blood through a circular tube with magnetic properties," Journal of Magnetism and Magnetic Materials, vol. 477, pp. 382-387, 2019.

[22] N. Ali Shah, D. Vieru, and C. Fetecau, "Effects of the fractional order and magnetic field on the blood flow in cylindrical domains," Journal of Magnetism and Magnetic Materials, vol. 409, pp. 10-19, 2016.

[23] J. C. Misra, A. Sinha, and G. C. Shit, "Mathematical modeling of blood flow in a porous vessel having double stenoses in the presence of an external magnetic field," International Journal of Biomathematics, vol. 4, no. 2, pp. 207-225, 2011.

[24] G. Ramamurthy and B. Shanker, "Magnetohydrodynamic effects on blood flow through a porous channel," Medical \& Biological Engineering \& Computing, vol. 32, no. 6, pp. 655659, 1994.

[25] I. M. Eldesoky and A. A. Mousa, "Peristaltic flow of a compressible non-Newtonian Maxwellian fluid through porous medium in a tube," International Journal of Biomathematics, vol. 3, no. 2, pp. 255-275, 2010.

[26] I. M. Eldesoky, "Slip effects on the unsteady MHD pulsatile blood flow through porous medium in an artery under the effect of body acceleration," International Journal of Mathematics and Mathematical Sciences, vol. 2012, Article ID 860239, 26 pages, 2012.

[27] K. Hosseinzadeh, S. Salehi, M. R. Mardani, F. Y. Mahmoudi, M. Waqas, and D. D. Ganji, "Investigation of nanoBioconvective fluid motile microorganism and nanoparticle flow by considering MHD and thermal radiation," Informatics in Medicine Unlocked, vol. 21, article 100462, 2020.

[28] K. Hosseinzadeh, S. Roghani, A. R. Mogharrebi, A. Asadi, and D. D. Ganji, "Optimization of hybrid nanoparticles with mixture fluid flow in an octagonal porous medium by effect of radiation and magnetic field," Journal of Thermal Analysis and Calorimetry, vol. 143, no. 2, pp. 1413-1424, 2021.

[29] M. Inc and A. Akgul, "Approximate solutions for MHD squeezing fluid flow by a novel method," Boundary Value Problems, vol. 2014, no. 1, Article ID 18, 2014.

[30] J. V. Ramana Reddy, D. Srikanth, and S. V. S. S. N. V. G. Krishna Murthy, "Mathematical modelling of pulsatile flow of blood through catheterized unsymmetric stenosed arteryEffects of tapering angle and slip velocity," European Journal of Mechanics - B/Fluids, vol. 48, pp. 236-244, 2014.

[31] A. Akgul, D. Baleanu, and M. Inc, "On the solutions of electrohydrodynamic flow with fractional diffierential equations by reproducing kernel method," Open Physics, vol. 128, pp. 218223, 2017.

[32] R. Hilfer, Threefold Introduction to Fractional Derivatives: In Anomalous Transport, Wiley-VCHVerlag Germany, 2008.

[33] N. A. Shah, T. Elnaqeeb, and S. Wang, "Effects of Dufour and fractional derivative on unsteady natural convection flow over an infinite vertical plate with constant heat and mass fluxes," Computational and Applied Mathematics, vol. 37, no. 4, pp. 4931-4943, 2018.

[34] N. A. Shah, T. Elnaqeeb, I. L. Animasaun, and Y. Mahsud, "Insight into the natural convection flow through a vertical cylinder using Caputo time-fractional derivatives," International Journal of Applied and Computational Mathematics, vol. 4 , no. 3, p. 80, 2018. 
[35] H. Stehfest, "Algorithm 368: numerical inversion of Laplace transforms [D5]," Communications of the ACM, vol. 13, no. 1, pp. 47-49, 1970.

[36] D. Y. Tzou, Macro to Micro Scale Heat Transfer: The Lagging Behavior, Taylor and Francis, Washington, 1997.

[37] S. S. Ardahaie, A. J. Amiri, A. Amouei, K. Hosseinzadeh, and D. D. Ganji, "Investigating the effect of adding nanoparticles to the blood flow in presence of magnetic field in a porous blood arterial," Informatics in Medicine Unlocked, vol. 10, pp. 71-81, 2018.

[38] M. Gholinia, K. Hosseinzadeh, and D. D. Ganji, "Investigation of different base fluids suspend by CNTs hybrid nanoparticle over a vertical circular cylinder with sinusoidal radius," Case Studies in Thermal Engineering, vol. 21, p. 100666, 2020.

[39] A. Jaishankar and G. H. McKinley, "Power-law rheology in the bulk and at the interface: quasi-properties and fractional constitutive equations," Proceedings of the Royal Society A: Mathematical, Physical and Engineering Sciences, vol. 469, no. 2149, article 20120284, 2012.

[40] M. Partohaghighi, M. Inc, M. Bayram, and D. Baleanu, "On numerical solution of the time fractional advection-diffusion equation involving atangana-baleanu-caputo derivative," Open Physics, vol. 17, no. 1, pp. 816-822, 2019.

[41] C. F. Lorenzo and T. Hartley, Generalized Functions for the Fractional Calculus, NASA/TP-1999-209424/REV1, 1999.

[42] H. Sheng, Y. Li, and Y. Q. Chen, "Application of numerical inverse Laplace transform algorithms in fractional calculus," Journal of the Franklin Institute, vol. 348, no. 2, pp. 315-330, 2011.

[43] D. K. Tong, X. Zhang, and X. Zhang, "Unsteady helical flows of a generalized Oldroyd-B fluid," Journal of Non-Newtonian Fluid Mechanics, vol. 156, no. 1-2, pp. 75-83, 2009.

[44] Y. Jiang, H. Qi, H. Xu, and X. Jiang, “Transient electroosmotic slip flow of fractional Oldroyd-B fluids7," Microfluidics and Nanofluidics, vol. 21, no. 1, 2017.

[45] I. L. Animasaun and I. Pop, "Numerical exploration of a nonNewtonian Carreau fluid flow driven by catalytic surface reactions on an upper horizontal surface of a paraboloid of revolution, buoyancy and stretching at the free stream," Alexandria Engineering Journal, vol. 56, no. 4, pp. 647-658, 2017.

[46] N. A. Shah, A. A. Zafar, and C. Fetecau, "Free convection flows over a vertical plate that applies shear stress to a fractional viscous fluid," Alexandria Engineering Journal, vol. 57, no. 4, pp. 2529-2540, 2017. 